Supplementary Materials

\title{
Role of electronic structures and dispersion interactions in adsorption selectivity of pyrimidine molecules with a $\operatorname{Si}\left(\begin{array}{lll}5 & 5 & 12\end{array}\right)$ surface
}

\author{
G-H. Kim, ${ }^{1}$ S Jeong, ${ }^{1, *}$ \\ ${ }^{1}$ Department of Physics, Chonbuk National University, Jeonju 54896, Korea \\ I. Lee, ${ }^{2}$ Md. A. Hanif, ${ }^{2}$ Md. A. Islam, ${ }^{2}$ K. P. Sapkota, ${ }^{2}$ J. R. Hahn ${ }^{2,3,}$, \\ ${ }^{2}$ Department of Chemistry, Chonbuk National University, Jeonju 54896, Korea \\ ${ }^{3}$ Textile Engineering, Chemistry and Science, North Carolina State University 2401 Research Dr. Raleigh, \\ NC 27695-8301, USA \\ *Corresponding authors
}

\section{Experimental Methods}

The $n$-type $\operatorname{Si}(5512)$ substrates ( $P$-doped, $0.1-10 \Omega \mathrm{cm}, 0.5 \mathrm{~mm}$ thick, Virginia Semiconductor) used in the present work were rinsed in an ultrasonic ethanol solution. The Si substrates were then degassed at $700^{\circ} \mathrm{C}$ for $24 \mathrm{~h}$ under ultra-high vacuum $\left(\sim 1 \times 10^{-10}\right.$ Torr $)$, sputtered by $1 \mathrm{keV} \mathrm{Ne}$ ions, and flash annealed at $1500 \mathrm{~K}$ for $15 \mathrm{~s}$. After flash annealing, the substrates were quickly cooled to $1200 \mathrm{~K}$ and then cooled to room temperature at a rate of $\sim 2 \mathrm{~K} / \mathrm{s}$ to induce surface reconstruction. The cleanliness of the substrates was examined using a homemade variable-low-temperature STM system. ${ }^{\mathrm{S} 1}$ The base pressure of the STM chamber was maintained $\sim 1.6 \times 10^{-11}$ Torr during STM operation. Liquid pyrimidine $(98 \%$, Sigma-Aldrich) was placed in a glass container connected to a vacuum leak valve installed on the STM chamber. Freeze-pump-thaw cycles were carried out to purify the liquid prior to dosing. The cleanliness and ordering of the $\operatorname{Si}(55$ 12) were examined by STM imaging. After clean surfaces were imaged, the STM tip was withdrawn for dosing of the pyrimidine. 
Pyrimidine dosing was carried out through a leak valve. The leak valve was connected to a microcapillary array-filled glass tube for uniform adsorption onto the surface. Electrochemically etched tungsten tips were cleaned and sharpened by field-emission selfsputtering in a Ne atmosphere and heating in a strong electric field. The STM tip was gently touched to the Si surface or treated by field emission in situ until the sharp step edges of the surface were confirmed.

\section{Theoretical Methods}

The binding configurations of the pyrimidines on the $\mathrm{Si}(5512)-2 \_1$ surface were calculated using the Vienna ab initio package $(\mathrm{VASP})^{\mathrm{S} 2}$ with projector-augmented waves $(\mathrm{PAWs})^{\mathrm{S} 3}$ and the generalized gradient approximation (GGA). ${ }^{\mathrm{S} 4}$ The surface was simulated using a repeated slab model that includes four Si layers with thickness of about $14 \AA$ and a $15.0 \AA$ vacuum layer. The bottom of the slab had a bulk-like structure in which each $\mathrm{Si}$ atom

was saturated by $\mathrm{H}$ atom(s). The central subunit $(D 2)^{\mathrm{S} 5}$ was used as the substrate because the entire (5 5 12) surface was too large to simulate. The super cell was $15.43 \AA$ along the $[1 \overline{1} 0]$ direction and $22.16 \AA$ along the $[66 \overline{5}]$ direction. A 30 Ry cutoff energy in the plane-wave basis and the gamma point in the surface Brillouin zone (SBZ) were used. The geometries of all atoms except the bottom $\mathrm{H}$ and $\mathrm{Si}$ atoms were optimized until the remaining forces acting on each ion were less than $0.02 \mathrm{eV} / \AA$. The present calculation parameters were sufficient to produce convergent results. ${ }^{\mathrm{S}, \mathrm{S} 6} \mathrm{With}$ inclusion of one more Si layer, the adsorption energies change from 2.01, 1.57, 1.41, and $1.49 \mathrm{eV}$ to $2.06,1.62,1.44$, and $1.53 \mathrm{eV}$ for Pym_ $\mathrm{A}_{1}$, $\mathrm{Pym}_{-} \mathrm{D}_{2}, \mathrm{Pym}_{-} \mathrm{T}_{2}$, and $\mathrm{Pym}_{-} \mathrm{H}_{1}$, respectively. The adsorption energies themselves increase by about $0.05 \mathrm{eV}$, but relative differences are within $0.01 \mathrm{eV}$. The STM images were simulated using the Tersoff-Hamann theory, in which tunneling current is proportional to the local density of states (LDOS) within the tunneling voltage and Fermi energy. ${ }^{\text {S7 }}$ 


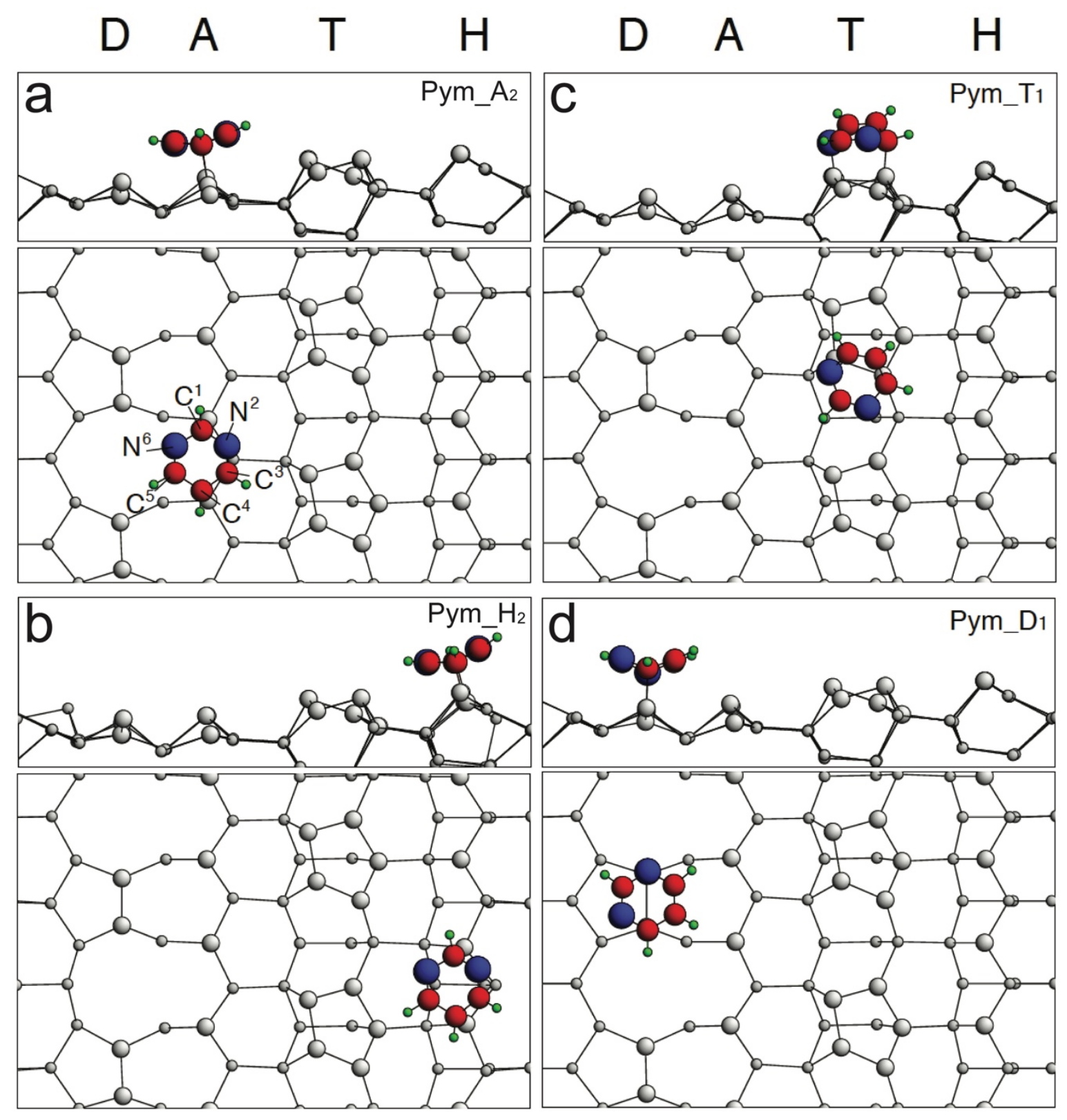

Figure S1 (color). Binding configurations of a pyrimidine molecule on the D2 subunits of $\operatorname{Si}(55$ 12) $-2 \times 1$. Top and side views of the stable structures of the pyrimidine molecule on A, D, T, and H positions [(a) Pym_A2, (b) Pym_H P $_{2}$ (c) Pym_T above the figure indicate the $\operatorname{Dmr}(\mathrm{D}), \operatorname{Adr}(\mathrm{A}), \operatorname{Tet}(\mathrm{T})$, and the $H n \mathrm{c}(\mathrm{H})$ sites. $\mathrm{C}^{1,3,4,5}$ and $\mathrm{N}^{2,6}$ characters in (b) are the numbered $\mathrm{C}$ and $\mathrm{N}$ atoms in the pyrimidine molecule. 

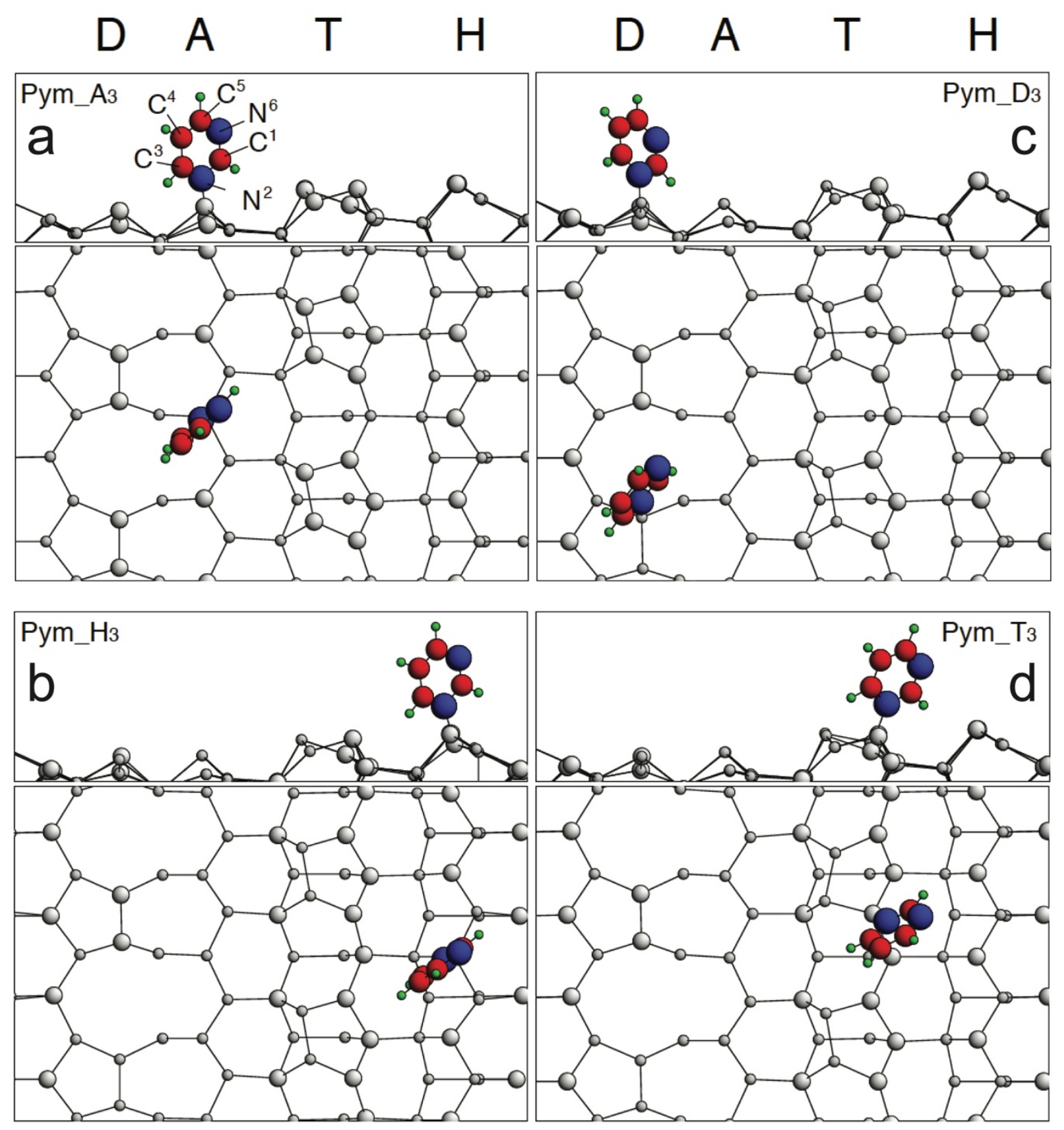

Figure S2 (color). Binding configurations of a pyrimidine molecule on the D2 subunits of $\operatorname{Si}(55$ 12) $-2 \times 1$. Top and side views of the stable structures of the pyrimidine molecule on A, D, T, and H positions [(a) Pym_A $A_{3}$, (b) $\mathrm{Pym}_{-} \mathrm{H}_{3}$, (c) Pym_ $\mathrm{D}_{3}$, and (d) Pym_T $\mathrm{T}_{3}$. The letters above the figure indicate the $\operatorname{Dmr}(\mathrm{D}), \operatorname{Adr}(\mathrm{A}), \operatorname{Tet}(\mathrm{T})$, and the $H n \mathrm{c}(\mathrm{H})$ sites. $\mathrm{C}^{1,3,4,5}$ and $\mathrm{N}^{2,6}$ in (b) are the numbered $\mathrm{C}$ and $\mathrm{N}$ atoms in the pyrimidine molecule. 


\section{Benzene}

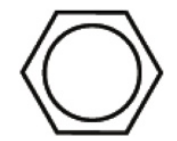

$+\mathrm{H}_{2}$ 1,4-cyclohexadiene

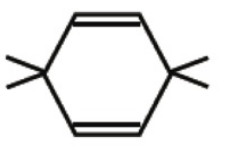

Process 1

\section{Surface}

$$
\mathrm{E}_{\mathrm{mol}}+\mathrm{E}_{\mathrm{sub}}+\mathrm{E}_{\mathrm{H}_{2}}
$$

Parallel

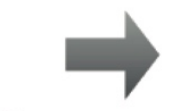

Process 2

Surface

Surface
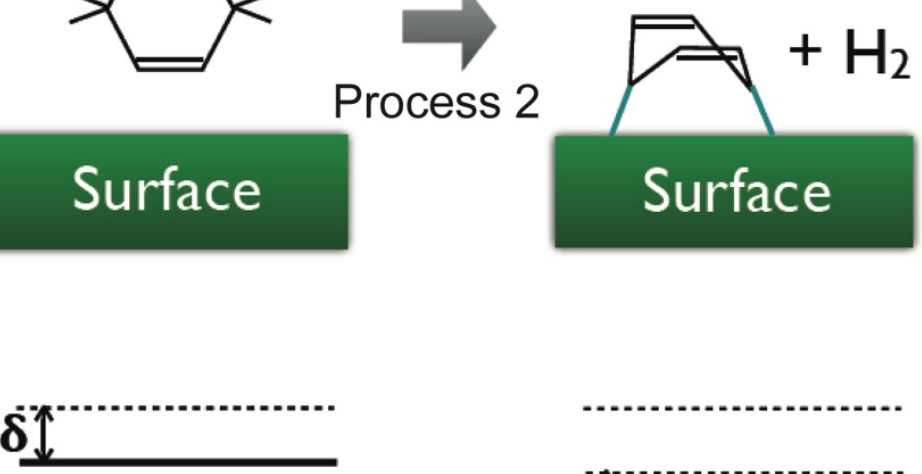

$\boldsymbol{\delta}=$ =hydrogenation energy

Figure S3 (color). Virtual processes and energy diagrams for benzene reacted with the $\operatorname{Si}(5$ 5 12)-2×1. Benzene combines with $\mathrm{H}_{2}$ to form 1,4-cyclohexadiene (Process 1), and 1,4cyclohexadiene binds to the surface with the release of $\mathrm{H}_{2}$ (Process 2). The energy changes during Processes 1 and 2 are indicated by $\delta$ (the hydrogenation energy) and $E_{\mathrm{b}}$ (the binding energy). 


\section{Supplementary References}

(S1) Hahn, J. R. Imaging and Manipulation of Benzene Molecules on Si Surfaces Using a Variable-Low Temperature Scanning Tunneling Microscope. Bull. Korean Chem. Soc. 2005, 26, 1071-1074.

(S2) Kresse, G.; Hafner, J. Ab initio Molecular Dynamics for Liquid Metals. Phys. Rev. B 1993, 47, R558-R561.

(S3) Blöchl, P. E. Projector Augmented-Wave Method. Phys. Rev. B 1994, 50, 17953-17979.

(S4) Perdew, J. P.; Burke, K.; Ernzerhof, M. Generalized Gradient Approximation Made Simple. Phys. Rev. Lett. 1996, 77, 3865-3868.

(S5) Jeong, S.; Jeong, H.; Cho, S.; Seo, J. M. New Structural Model of the High-Index Si(5 5 12)-2×1. Surf. Sci. 2004, 557, 183-189.

(S6) Jeong, H.; Jeong, S.; Jang, S. H.; Seo, J. M.; Hahn, J. R. Atomic Structures of Benzene and Pyridine on Si(5 5 12)-2×1. J. Phys. Chem. B 2006, 110, 15912-15919.

(S7) Tersoff, J.; Hamann, D. R. Theory and Application for the Scanning Tunneling Microscope. Phys. Rev. Lett. 1983, 50, 1998-2001. 